Check for updates

Cite this: RSC Adv., 2019, 9, 730

Received 2nd October 2018

Accepted 12th December 2018

DOI: $10.1039 / c 8 r a 08173 e$

rsc.li/rsc-advances

\section{Facile organic surfactant removal of various dimensionality nanomaterials using low- temperature photochemical treatment $\uparrow$}

\author{
Chahwan Hwang,,$^{\text {a }}$ Jae Sang Heo, ${ }^{\text {bc }}$ Kyung-Tae Kim, ${ }^{\text {b }}$ Yeo Kyung Kang, ${ }^{a}$ \\ Byungdoo Choi, ${ }^{a}$ Yong-Hoon Kim, (DD d Antonio Facchetti, ${ }^{\text {ef }}$ Sung Kyu Park (DD *b \\ and Myung-Gil Kim (D)*a
}

\begin{abstract}
Deep ultraviolet (DUV)-treatment is an efficient method for the removal of high-energy-barrier polymeric or aliphatic organic ligands from nanomaterials. Regardless of morphology and material, the treatment can be used for nanoparticles, nanowires, and even nanosheets. The high-energy photon irradiation from lowpressure mercury lamps or radio frequency (RF) discharge excimer lamps could enhance the electrical conductivity of various nanomaterial matrixes, such as $\mathrm{Ag}$ nanoparticles, $\mathrm{Bi}_{2} \mathrm{Se}_{3}$ nanosheets, and $\mathrm{Ag}$ nanowires, with the aliphatic alkyl chained ligand (oleylamine; OAm) and polymeric ligand (polyvinyl pyrrolidone; PVP) as surfactants. In particular, Ag nanoparticles (AgNPs) that are DUV-treated with polyvinyl pyrrolidone (PVP) for $90 \mathrm{~min}\left(50-60{ }^{\circ} \mathrm{C}\right.$ ) exhibited a sheet resistance of $0.54 \Omega \square^{-1}$, while thermal-treated AgNP with PVP had a sheet resistance of $7.5 \mathrm{k} \Omega \square^{-1}$ at $60{ }^{\circ} \mathrm{C}$. The simple photochemical treatment on various dimensionality nanomaterials will be an efficient sintering method for flexible devices and wearable devices with solution-processed nanomaterials.
\end{abstract}

\section{Introduction}

Solution-processed electronics are able to achieve high throughput, material efficiency, and cost-effective fabrication for next-generation large area electronics, such as flexible displays, wearable devices, and electronic skins. ${ }^{1-6}$ Novel materials and innovative processing methods have been investigated for their applicability as low-temperature solutionprocessed electronic devices with high electrical performance and unprecedented functionalities. ${ }^{7,8}$ For example, the variety of chemical groups in organic semiconductors enables the simple control of chemical properties for solution processing and good electrical performance. ${ }^{\mathbf{9}, 10}$ Moreover, there have been significant efforts on newly emerging inorganic materials, such as sol-gel

\footnotetext{
${ }^{a}$ Department of Chemistry, Chung-Ang University, Seoul 06980, Korea. E-mail: myunggil@cau.ac.kr

${ }^{b}$ School of Electrical and Electronics Engineering, Chung-Ang University, Seoul 06980, Korea.E-mail: skpark@cau.ac.kr

'Department of Medicine, University of Connecticut Health Center, Farmington, CT 06030, USA

${ }^{d}$ SKKU Advanced Institute of Nanotechnology (SAINT), School of Advanced Materials Science and Engineering, Sungkyunkwan University, Suwon 16419, Korea

${ }^{e}$ Department of Chemistry, The Materials Research Center, Northwestern University, 2145 Sheridan Road, Evanston, Illinois 60208, USA

${ }^{f}$ Flexterra Corporation, 8025 Lamon Avenue, Skokie, Illinois 60077, USA

$\dagger$ Electronic supplementary information (ESI) available: [DETAILS]. See DOI: 10.1039/c8ra08173e

\$ These two authors contributed equally to this work and are co-first authors.
}

oxide precursors, soluble metal halide perovskite, nanomaterials, and polymeric silicon precursors, which provide soluble materials or precursors with intrinsically high electrical performances. ${ }^{11-14}$ In particular, soluble inorganic nanomaterials exhibited promising electronic functionalities, which include high electrical conductivity with optical transparency, high carrier mobility, high optical absorption, or chemical sensing abilities. ${ }^{15,16}$ The well-developed chemical synthetic strategies of nanomaterials enabled finely tuned electronic materials for each target application, which surpasses even the corresponding bulk material properties. ${ }^{17,18}$

The incorporation of organic ligands, such as polyvinyl pyrrolidone (PVP), oleylamine (OAm), oleic acid (OA), and polyamidoamine (PAMAM), is important to achieving highly soluble nanomaterials. ${ }^{19-21}$ Although the use of organic ligands as a surfactant could stabilize high-energy surfaces and prevent the aggregation of nanomaterials, ${ }^{20}$ the long-chain organic ligands on nanomaterial surfaces can act as highly electric insulating barriers between nanomaterials, which results in the poor electrical connection of nanomaterials and significant electrical performance degradation. ${ }^{22}$ To improve the electrical performance of nanomaterial networks, the removal of organic ligands is a crucial step for the intimate contact formation between nanomaterials and subsequent fast-charge transport in nanomaterial matrixes. Until now, several strategies have been developed to remove the organic ligands with insulating characteristics using thermal annealing, UV-ozone (UVO) treatment, or the ligand-exchange method. ${ }^{22-24}$ of these strategies, high- 
temperature thermal annealing under inert, oxidative, or reductive atmospheres has been widely employed as a simple and effective method. ${ }^{23,24}$ Unfortunately, the thermal annealing method results in undesirable morphological changes, such as grain growth and the destruction of anisotropic shapes, the loss of the quantum confinement effect, and electric disconnection. ${ }^{25,26}$ Moreover, high-temperature treatment is incompatible with flexible plastic substrates such as polyethylene terephthalate (PET) ${ }^{27}$ In the case of UVO treatment, the low thermal budget ligand removal is possible with high oxidative $\mathrm{O}_{3}$ generation. Although $\mathrm{O}_{3}$ could easily oxidize and remove organic ligands, undesirable oxidation on nanomaterial surfaces results in significant electrical performance degradation. ${ }^{28}$ Unlike thermal annealing and UVO treatment, the ligand-exchange strategy can convert long alkyl chains on nanomaterials to short organic molecules that can be easily removed with mild heating or low-temperature vacuum treatment. ${ }^{29-31}$ However, the ligand-exchange method is only applicable for alkyl chained-ligands with relatively low binding strengths on nanomaterials. In addition, there is organic acid treatment or hydrazine treatment for the cleaning of nanomaterial surfaces, which generally induces the aggregation of nanomaterials or produces potentially hazardous waste. ${ }^{32,33}$

For organic and inorganic materials, there is a significant contrast in terms of their photochemical stabilities. ${ }^{34,35}$ While the weak covalent bonding in organic molecules can be destructed by high-intensity deep ultraviolet (DUV) light irradiation, relatively stable inorganic materials with extended structures usually remain intact, even after high-dose DUV irradiation. ${ }^{36}$ For example, high-quality metal oxide semiconductor (MOS) films and well-defined organic semiconductor films were successfully demonstrated by using photochemical annealing and photochemical patterning, respectively. ${ }^{34,35}$ Considering the photochemical stability difference between organic ligands and inorganic nanomaterials, the simple DUV irradiation could successfully achieve organic surfactant removal on inorganic nanomaterials, and enhance the charge transport between nanomaterials.

In this study, we report that the simple photochemical DUVtreatment on solution-processed nanomaterial matrixes achieved facile removal of organic surfactants and significant charge-transport enhancement between nanomaterials. To compare the thermal annealing method and DUV treatment at low temperature, two types of ligands on $\mathrm{Ag}$ nanoparticles (AgNPs), namely the aliphatic alkyl chained ligand (oleylamine; OAm) and polymeric ligand (polyvinyl pyrrolidone; PVP), were used as surfactants. The DUV treatment for these materials showed reasonable or even superior electrical performance compared with the thermal annealing method without any change of shape and aggregation. Furthermore, we investigated the effect of low-temperature photochemical treatment for various dimensional conducting nanomaterials such as $\mathrm{Bi}_{2} \mathrm{Se}_{3}$ nanosheets (NSs), and Ag nanowires (NWs). Overall, the DUV treatment could be employed as a general strategy for the lowtemperature electrical activation of nanomaterials for nextgeneration low-cost large-area electronics.

\section{Experimental}

\section{Nanomaterial synthesis}

Reagents. All reagents were purchased from Sigma-Aldrich and were used without further purification.

PVP-capped Ag nanowires. PVP-capped AgNWs (PVP-AgNWs) were synthesized using the polyol process. ${ }^{37}$ Typically, $0.667 \mathrm{~g}$ of polyvinylpyrrolidone (PVP) (MW 58 000) was dissolved in $200 \mathrm{ml}$ of ethyleneglycol (EG), after which $6 \mathrm{ml}$ of $\mathrm{NiCl}_{2}$ (1 mM in EG) solution was added as a seed molecule above the solution with stirring at $400 \mathrm{rpm}$. After the reaction temperature of the solution was increased to $170{ }^{\circ} \mathrm{C}, 5.1 \mathrm{~g}$ of $\mathrm{AgNO}_{3}$ solution (10 wt\% in $\mathrm{EG)}$ ) was added dropwise into the solution. The reaction was completed after $1 \mathrm{~h}$, and the solution was cooled to room temperature. Then, $600 \mathrm{ml}$ of acetone was added to the solution to precipitate the AgNWs, after which the supernatant was decanted. The precipitates were dispersed in $50 \mathrm{ml}$ of isopropyl alcohol (IPA), and then the dispersion was centrifuged at $4000 \mathrm{rpm}$ for $3 \mathrm{~min}$. The process was repeated 3 times. Finally, the AgNWs were dispersed in IPA for $0.5 \mathrm{wt} \%$ prior to spray coating.

PVP-capped Ag nanoparticles. PVP-capped AgNPs (PVPAgNPs) were synthesized using the simple solution reduction method. ${ }^{38}$ Typically, $4.25 \mathrm{~g}$ of $\mathrm{AgNO}_{3}$ and $4.2 \mathrm{~g}$ of polyvinylpyrrolidone (MW 58 000) was dissolved in $400 \mathrm{ml}$ of ethyleneglycol. The solution temperature was increased to $80{ }^{\circ} \mathrm{C}$. After the dissolution of all reagents, $3 \mathrm{ml}$ of formic acid (85\%) was slowly dropped into the solution, and then the solution was further stirred for $12 \mathrm{~h}$. The solution was cooled, and $1200 \mathrm{ml}$ of acetone was added to the solution to precipitate the AgNPs. After the AgNPs completely precipitated, the supernatant was decanted. Then, $100 \mathrm{ml}$ of hot de-ionized water/IPA (1:1) solution was added to the precipitates and sonicated for $30 \mathrm{~min}$ to fully dispersed AgNPs. Next, $300 \mathrm{ml}$ of acetone was added to the dispersion, and it was then centrifuged at $5000 \mathrm{rpm}$ for $3 \mathrm{~min}$. The process was repeated 5 times. Finally, the PVP-AgNPs were dispersed in IPA for $25 \mathrm{wt} \%$ with sonication.

Oleylamine-capped $\mathbf{A g}$ nanoparticles. Oleylamine-capped AgNPs (OAm-AgNPs) were synthesized as in previous literature. ${ }^{39}$ To do this, $1.02 \mathrm{~g}$ of $\mathrm{AgNO}_{3}$ and $1 \mathrm{ml}$ of 1 -octanol were mixed in $60 \mathrm{ml}$ of oleylamine, and the solution temperature was kept at $120{ }^{\circ} \mathrm{C}$ under vacuum for $30 \mathrm{~min}$ with stirring to dissolve all of the components and to remove the water molecules. Then, the reaction vessel was filled with Ar gas and the solution was heated at $160{ }^{\circ} \mathrm{C}$ for an additional $2 \mathrm{~h}$ until the reaction was finished. After the solution was cooled to room temperature, $180 \mathrm{ml}$ of acetone was poured into the solution to precipitate OAm-AgNPs, and then the supernatant was decanted. The precipitated OAm-AgNPs were redispersed with $20 \mathrm{ml}$ of toluene, and $30 \mathrm{ml}$ of acetone was added. The mixture was centrifuged at $7000 \mathrm{rpm}$ for $5 \mathrm{~min}$. The washing process (redispersion and precipitation) was repeated 3 times to obtain purified OAmAgNPs. Finally, the OAm-AgNPs were redispersed in toluene at $17.5 \mathrm{wt} \%$ to prepare an appropriate solution for spin coating.

PVP-capped $\mathrm{Bi}_{2} \mathrm{Se}_{3}$ nanosheets. PVP-capped $\mathrm{Bi}_{2} \mathrm{Se}_{3}$ NSs (PVP$\mathrm{Bi}_{2} \mathrm{Se}_{3} \mathrm{NSs}$ ) were synthesized as reported in previous literature. ${ }^{\mathbf{4 0}}$ To do this, $0.485 \mathrm{~g}$ of $\mathrm{Bi}\left(\mathrm{NO}_{3}\right)_{3} \cdot 5 \mathrm{H}_{2} \mathrm{O}, 0.259 \mathrm{~g}$ of $\mathrm{Na}_{2} \mathrm{SeO}_{3}$, and 
$1.11 \mathrm{~g}$ of polyvinylpyrrolidone (MW 58 000) were dissolved in $150 \mathrm{ml}$ of ethyleneglycol. Then, the solution was refluxed at $190{ }^{\circ} \mathrm{C}$ for $6 \mathrm{~h}$ with stirring. After the reaction was finished, the black solution was cooled to room temperature, and $300 \mathrm{ml}$ of acetone was added to the solution to obtain $\mathrm{Bi}_{2} \mathrm{Se}_{3}$ precipitates. The precipitates were redispersed with $40 \mathrm{ml}$ of IPA, and then centrifuged at $8000 \mathrm{rpm}$ for $7 \mathrm{~min}$. The process was then repeated 3 times to remove the impurities. After final centrifugation, $\mathrm{Bi}_{2} \mathrm{Se}_{3} \mathrm{NSs}$ were redispersed with $10 \mathrm{ml}$ of IPA. The $\mathrm{Bi}_{2} \mathrm{Se}_{3} \mathrm{NSs}$ dispersion was centrifuged at $3000 \mathrm{rpm}$ for $3 \mathrm{~min}$ prior to spray coating, and then the supernatant was used for the spray coating solution.

\section{Film deposition and characterization}

PVP- or OAm-capped AgNP thin films. Both PVP-AgNPs and OAm-AgNPs thin films were fabricated using the spin-coating method. A total of $0.2 \mathrm{ml}$ of AgNP solution was dropped onto the spinning $(2500 \mathrm{rpm})$ silicon wafer substrate. For thermal annealing, the AgNP films were heated on a hot plate. For the photochemical treatment, the AgNP films were irradiated with a low-pressure mercury lamp (UV-1, Samco Co.) or a radio frequency (RF) discharge flat excimer lamp (EX-mini L12530, Hamamatsu Photonics K.K.) under nitrogen atmosphere. As a typical DUV irradiation source, the low-pressure mercury lamp (LPML) emits $253.7 \mathrm{~nm}$ (90\%) and $184.9 \mathrm{~nm} \mathrm{(10 \% ),} \mathrm{with} \mathrm{an}$ output intensity of $18-23 \mathrm{~mW} \mathrm{~cm}{ }^{-2}$. For excimer treatment, the excimer lamp emits $172 \mathrm{~nm}$ with an output intensity of $50 \mathrm{~mW}$ $\mathrm{cm}^{-2}$.

Ag NW and $\mathrm{Bi}_{2} \mathrm{Se}_{3} \mathrm{NS}$ thin films. AgNWs and $\mathrm{Bi}_{2} \mathrm{Se}_{3} \mathrm{NS}$ thin films were prepared with spray coating on cleaned silicon wafer substrates using an air brush (ZECO). The AgNW dispersion was spray coated on the substrates at $110{ }^{\circ} \mathrm{C}$ with an air pressure of 1 atm. The $\mathrm{Bi}_{2} \mathrm{Se}_{3}$ NSs dispersion was also spray coated on the substrate under the same conditions. The as-prepared AgNWs and $\mathrm{Bi}_{2} \mathrm{Se}_{3} \mathrm{NS}$ thin films were thermally or photochemically treated.

Thin-film and electrical characterization. The scanning electron microscopy (SEM) images were obtained using a fieldemission scanning electron microscope (SIGMA, Carl Zeiss, Germany) at an accelerating voltage of $5 \mathrm{kV}$. The Raman spectra were obtained with LabRam Aramis (Horiba, Japan) at a $785 \mathrm{~nm}$ excitation wavelength. The surface elements on the nanomaterial films were determined by X-ray photoelectron spectroscopy (XPS, Thermo U. K. K-alpha) using monochromated Al $\mathrm{K} \alpha$. The sheet resistance of the nanomaterial films were characterized with an Agilent 4155C semiconductor parameter analyzer, using the Van der Pauw method.

\section{Results and discussion}

Fig. 1 schematically describes the photochemical removal process of organic ligands and the fabrication of conducting electrodes with nanomaterials. As shown in Fig. 1a, the nanomaterial conductors initially have a poor electrical connection between spatially separated nanomaterials with organic ligands on nanomaterial surfaces, regardless of the dimension or morphology of the nanomaterials, such as NPs, NWs, and NSs. Generally, the insulating organic ligands with a high-energy barrier hinder the carrier movement and simultaneously decrease the electrical conductivity of nanomaterial arrays. Therefore, it is essential that these insulating ligands should be removed using effective methods to form a carrier-transport pathway between nanomaterials. As shown in Fig. 1b, we expected that successful removal of the organic ligands on metal nanomaterials via photochemical activation (DUV or excimer treatment; $254 \mathrm{~nm} / 185 \mathrm{~nm}$ or $172 \mathrm{~nm}$ ) can lead to the bond breaking of organic surfactants with a sufficiently high photon energy and the easy percolation of free charge carriers between nanomaterials. Consequently, the photochemically treated nanomaterials could achieve higher electrical conductivity than nanomaterials without photochemical activation owing to the clean removal of insulating ligands on nanomaterials. In order to investigate the efficiency of photochemical treatment for the removal of organic ligands, we used two different types of ligand (OAm and PVP) capped AgNPs, and three types of morphology (0D, 1D, 2D) which correspond to PVP- or OAm-AgNPs, PVPAgNWs, and PVP-Bi ${ }_{2} \mathrm{Se}_{3}$ NSs, respectively.

Fig. 2 shows the sheet resistances of nanomaterials (AgNPs, AgNWs, $\mathrm{Bi}_{2} \mathrm{Se}_{3} \mathrm{NSs}$ ) with thermal and DUV-treatment. In the case of the AgNPs, aliphatic oleylamine (OAm) and polymeric polyvinylpyrrolidone (PVP) ligands were used as surfactants. The initial nanomaterials showed poor electrical conductivities with few available electrical contacts between nanomaterials or impurity mediated conduction. ${ }^{41}$ As shown in Fig. 2a and c, although the initial conductive characteristics were different, the sheet resistances of AgNP films with OAm and PVP decreased according to the increase of the annealing temperature (30 min). The thermal annealing induces the shrinkage, vaporization, or partial decomposition of polymeric or hydrocarbon chains, which make the surfaces of each AgNP have closer contact. Furthermore, the AgNPs that in contact were sintered at the specific temperature because of the lower melting point of small AgNPs with high surface energy. However, a relatively high temperature (above $200{ }^{\circ} \mathrm{C}$ ) is still needed to achieve higher conductivity values that are similar to that of bulk silver, so thermal annealing treatment is not suitable for the fabrication of conducting electrodes on cheap plastic substrates, such as PET film. Compared to thermal annealing treatment, DUV treatment significantly decreased the sheet resistance of AgNPs at low temperatures of 50-60 ${ }^{\circ} \mathrm{C}$. In particular, the DUV treatment of AgNPs with PVP for $30 \mathrm{~min}$ $\left(50-60{ }^{\circ} \mathrm{C}\right)$ resulted in a sheet resistance of $4.45 \Omega \square^{-1}$, while the thermal-treatment of AgNPs with PVP resulted in a sheet resistance of $7.5 \mathrm{k} \Omega \square^{-1}$ at a similar annealing temperature of $60{ }^{\circ} \mathrm{C}$. The further DUV irradiation for $90 \mathrm{~min}$ on AgNPs with PVP resulted in a very low sheet resistance of $0.54 \Omega \square^{-1}$ without film morphology degradation. This result indicates that the DUV treatment process induces a dramatic reduction in the sheet resistance of AgNP films at low temperature owing to the effective cleavage or removal of organic ligands on AgNP materials. ${ }^{34,35}$

To demonstrate the generality of photochemical treatment for low-temperature nanomaterial conductor fabrication, 


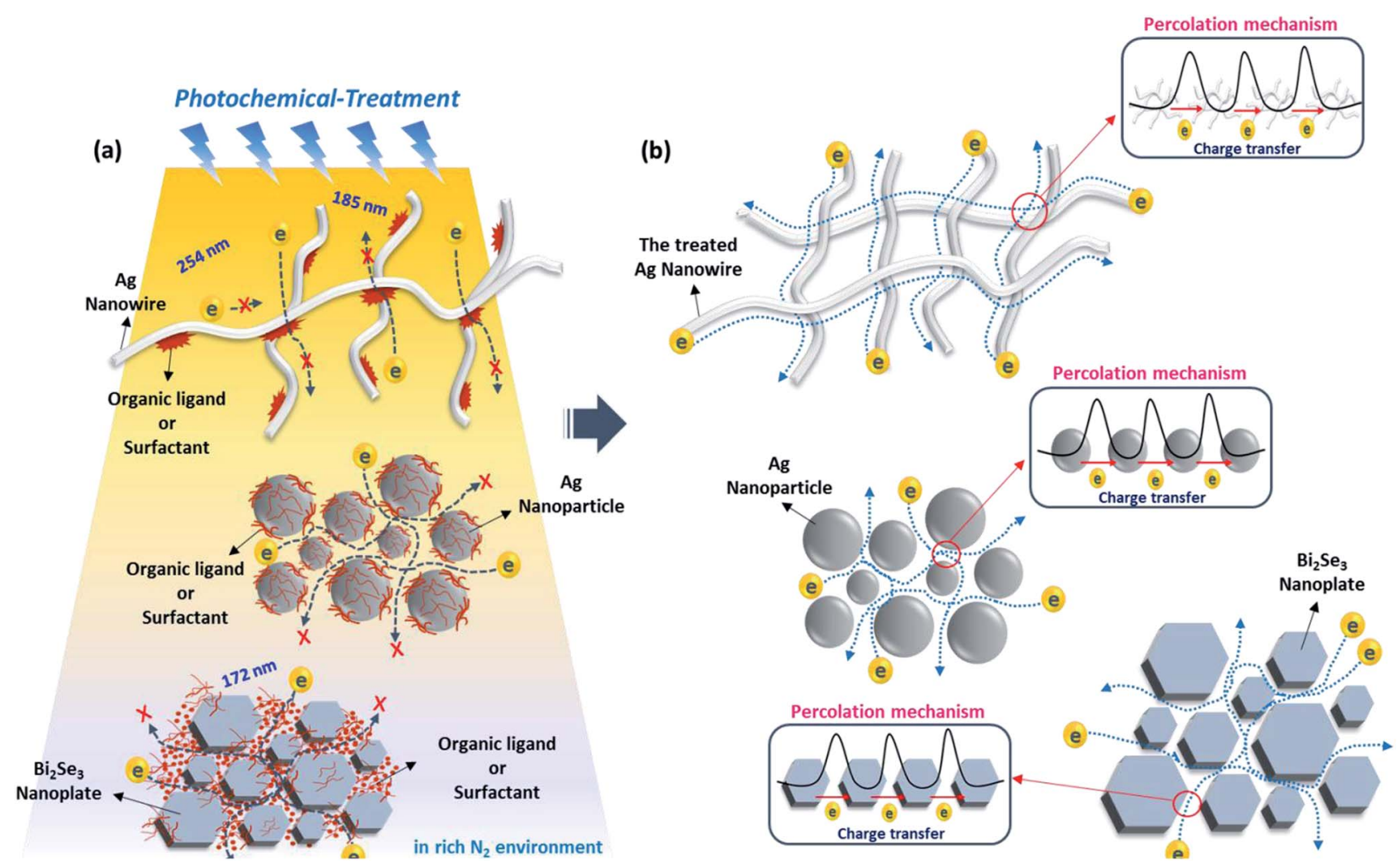

Fig. 1 Experimental scheme for photochemical activation on various dimensional metal or metal-chalcogenide nanomaterials. (a) Concept for photochemical activation and removal of organic ligands on nanomaterials. (b) Percolation mechanism of various morphology nanomaterials by photochemical activation.

nanomaterials having various shapes, such as PVP-AgNWs and $\mathrm{PVP}-\mathrm{Bi}_{2} \mathrm{Se}_{3}$ NSs films, were exposed to DUV and excimer (172 $\mathrm{nm})$ irradiations. The PVP-AgNW film exhibited a similar tendency as that of PVP-AgNP films for DUV treatment. The resistance of PVP-AgNW films reduced up to $16 \Omega \square^{-1}$ as the DUV exposure time increased (Fig. 2e). In comparison, the thermally treated AgNW films showed improved sheet resistance of $25.7 \Omega \square^{-1}$ after high temperature annealing at $150^{\circ} \mathrm{C}$ (ESI Fig. S1a $\uparrow$ ). However, PVP-Bi $\mathrm{Se}_{3}$ NS films could not achieve high conductivity close to the bulk characteristic using only DUV treatment, unlike PVP-AgNP or PVP-AgNW films. Without

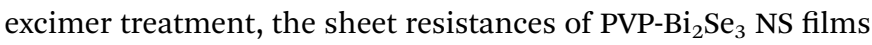
with DUV-60 min and DUV-120 min had values of $435 \mathrm{k} \Omega \square^{-1}$ and $175 \mathrm{k} \Omega \square^{-1}$, respectively. The sheet-shape of $\mathrm{PVP}-\mathrm{Bi}_{2} \mathrm{Se}_{3}$ NSs may reflect electromagnetic wave and hinder its pathway through the stacked-structure. The confined nature of organic ligands between $\mathrm{Bi}_{2} \mathrm{Se}_{3}$ NSs resulted the significant increase of thermal treatment temperature of the NS films upto $300{ }^{\circ} \mathrm{C}$ for reasonable sheet resistance of $473 \Omega \square^{-1}$ (ESI Fig. S1b $\dagger$ ). In contrast, shorter wavelength-excimer treatment $(30 \mathrm{~min})$ can lead to the enhancement of the conductivity of PVP- $\mathrm{Bi}_{2} \mathrm{Se}_{3} \mathrm{NS}$ films (49 k $\left.\Omega \square^{-1}\right)$. In the case where both DUV and excimer were sequentially treated in a short time, the PVP on $\mathrm{Bi}_{2} \mathrm{Se}_{3} \mathrm{NSs}$ was effectively removed. In our preliminary test, a long irradiation time of the excimer (>30 min) with high-intensity energy enables the removal of the organic ligands, and influences the structural integrity of PVP- $\mathrm{Bi}_{2} \mathrm{Se}_{3} \mathrm{NS}$, resulting in the degradation of the electrical performance. As shown in Fig. 2f, the sheet resistance of $\mathrm{PVP}-\mathrm{Bi}_{2} \mathrm{Se}_{3}$ the NS film significantly decreased to $45 \Omega \square^{-1}$ by the successive photochemical activation with two different values of photo-energy (DUV and excimer). Consequently, it indicated that photochemical activation is effectively capable of removing the insulating organic ligands on the surface of nanomaterials without any thermal treatment, regardless of the shape or structure of nanomaterials.

In order to investigate the variation of the OAm-AgNPs surface morphology with or without thermal or DUV treatment, field-emission scanning electron microscopy (FE-SEM) images were obtained, as shown in Fig. 3a-d. The pristine sample (Fig. 3a) showed that a large amount of OAm ligand is present within the large size of AgNPs and OAm aggregates. After thermal $\left(<150{ }^{\circ} \mathrm{C}\right)$ or DUV treatment, the OAm ligand aggregates on the top surface of AgNPs were removed, and the original grain of AgNPs was revealed owing mainly to OAm slow evaporation, as shown in Fig. 3c, d and S2 (ESI $\dagger$ ). However, the grains of OAm-AgNPs grew and aggregated above a specific annealing temperature $\left(>150{ }^{\circ} \mathrm{C}\right)$ owing to the filling of the empty space during the OAm evaporation and sintering (Fig. 3b). ${ }^{42}$ This aggregation phenomenon shifted toward the side edge of the substrate, which resulted in the generation of a charge-carrier transport pathway at the only side edge, as shown in Fig. S3 (ESI $\dagger$ ). On the contrary, with the increased 
(a)

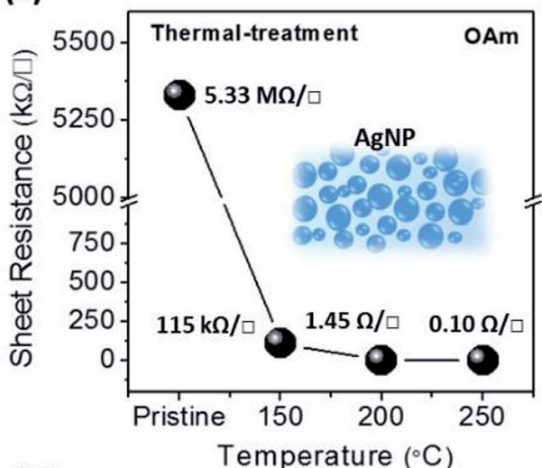

(b)

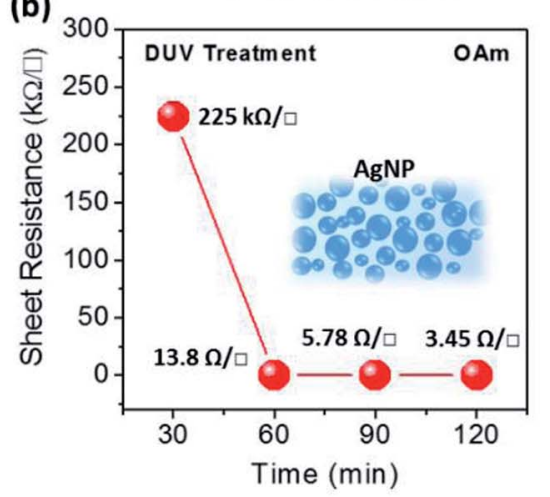

(c)

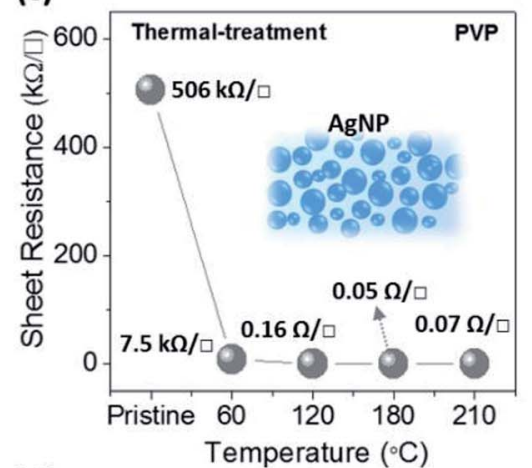

(d)

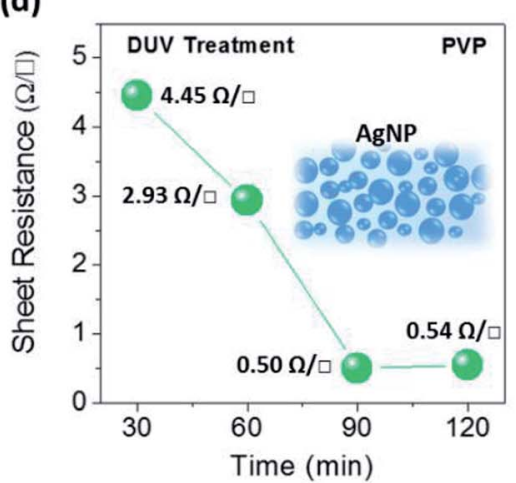

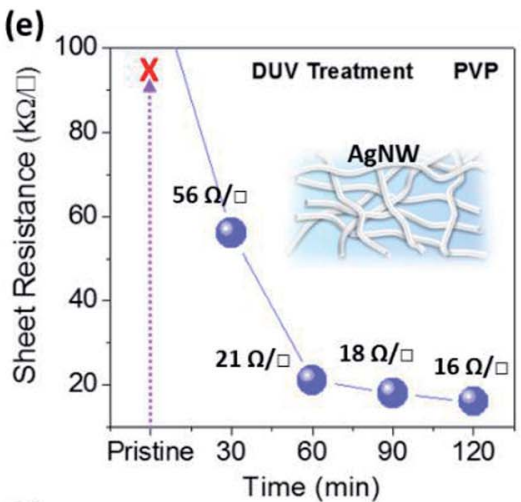

(f)

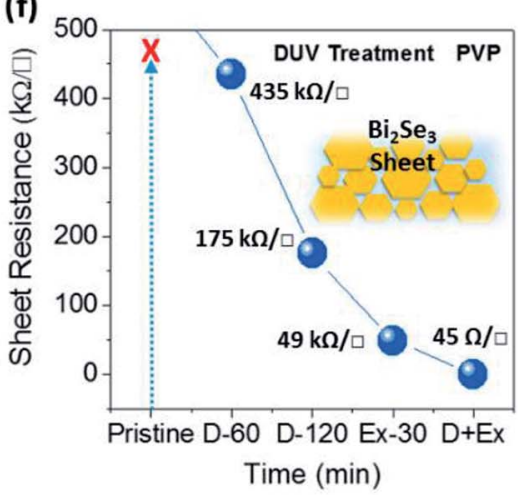

Fig. 2 Electrical characteristics of metal or metal-chalcogenide nanomaterials (AgNPs, AgNWs, and Bi $2 \mathrm{Se}_{3} \mathrm{NSs}$ ). (a) Sheet resistance of OAmAgNPs by thermal treatment at R.T., 150, 200, and $250^{\circ} \mathrm{C}$. (b) Sheet resistance of OAm-AgNPs by DUV treatment for 30, 60, 90, 120 min. (c) Sheet resistance of PVP-AgNPs by thermal treatment at R.T., 60, 120, 180 and $210^{\circ} \mathrm{C}$. (d) Sheet resistance of PVP-AgNPs by DUV treatment for 30,60 , 90, 120 min. (e) Sheet resistance of PVP-AgNWs by DUV treatment for 30, 60, 90, $120 \mathrm{~min}$. (f) $\mathrm{Sheet}$ resistance of PVP-Bi $\mathrm{Se}_{3} \mathrm{NSs}_{\mathrm{s}}$ by DUV treatment for 60, $120 \mathrm{~min}$, excimer only for $30 \mathrm{~min}$, and DUV $30 \mathrm{~min}+$ excimer $30 \mathrm{~min}$.

irradiation time of DUV treatment, there was no significant variation of the size or shape and aggregation of AgNPs. The DUV treatment induced the slight growth of the particle size with an increasing DUV irradiation time because the partial nano-welding between particles that were in close contact was due to the plasmon effect (Fig. S4 in ESI $\dagger$ ). ${ }^{43,44}$ Consequently, the
FE-SEM images showed that the photochemical treatment is an effective process for the removal of OAm ligands on AgNPs without any deformation or degradation of AgNPs in spite of the long irradiation time at low temperature. To confirm the OAm ligand removal on AgNP surfaces using DUV treatment, we have implemented Raman spectroscopy analysis for OAm-capped
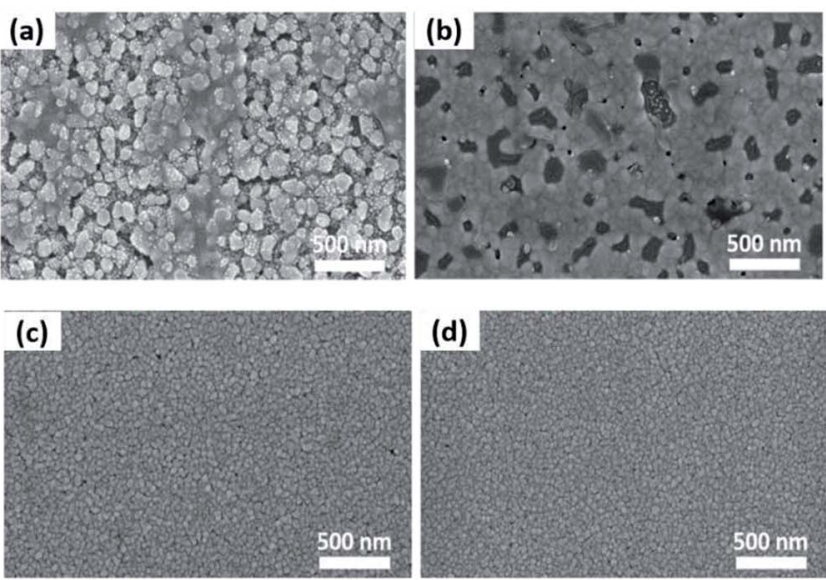
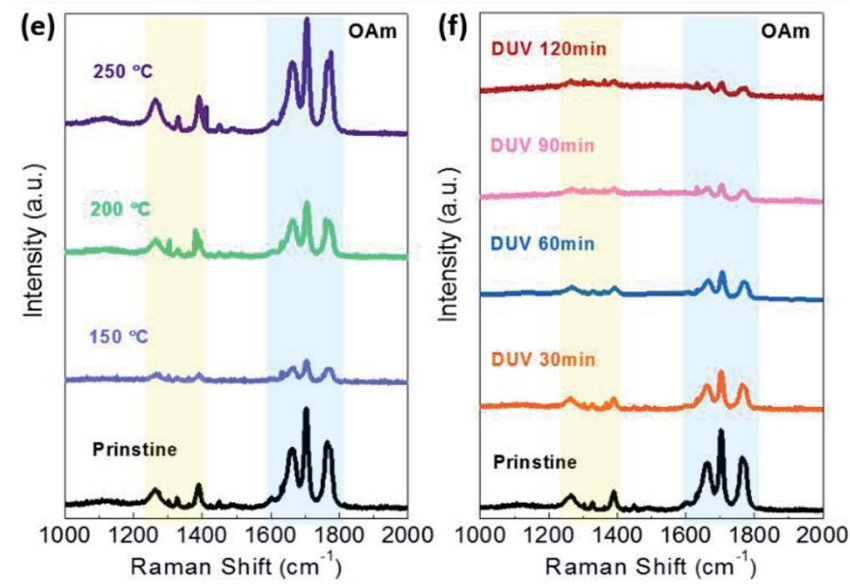

Fig. 3 Morphology (FE-SEM) and chemical analysis (Raman) of the OAm-AgNPs with DUV and thermal treatment. (a and b) FESEM image of (a) pristine OAm-AgNPs, and (b) thermal annealed OAm-AgNPs at $200^{\circ} \mathrm{C}$. (c and d) FESEM image of DUV treated OAm-AgNPs for (c) 60 min and (d) 90 min. (e and f) Raman spectrum variation of OAm-AgNP films obtained using (e) thermal and (f) DUV treatment. 

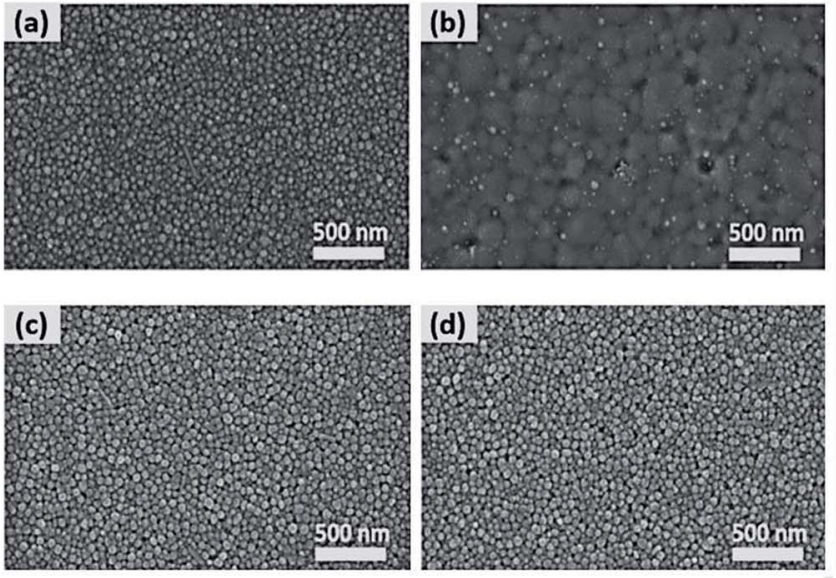
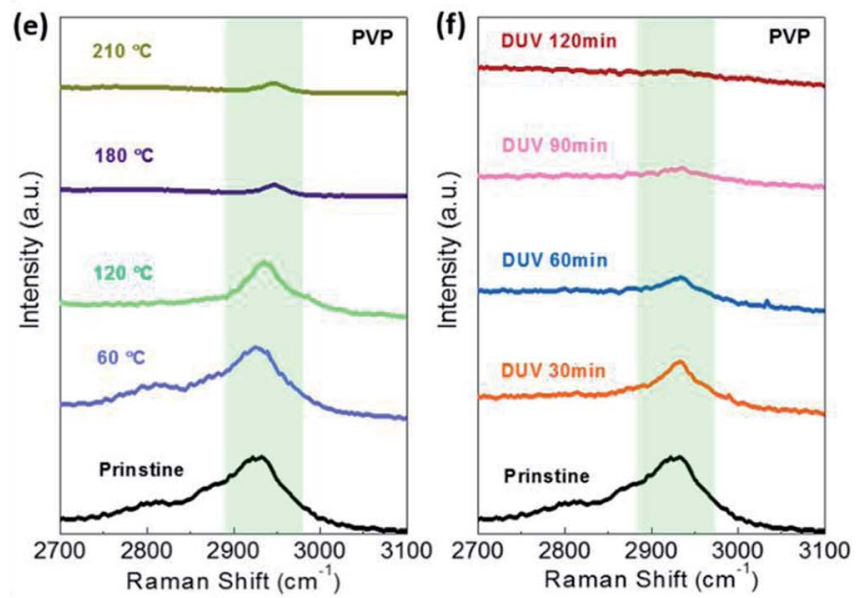

Fig. 4 Morphology (FE-SEM) and chemical analysis (Raman) of the PVP-AgNPs with DUV and thermal treatment. (a and b) FESEM image of (a) pristine PVP-AgNPs and (b) thermally annealed PVP-AgNPs at $180^{\circ} \mathrm{C}$. (c and d) FESEM image of DUV treated PVP-AgNPs for (c) 60 min and (d) 90 min. (e and f) Variation of Raman spectrum of PVP-AgNP films obtained via (e) thermal and (f) DUV treatment.

AgNP films with different DUV irradiation times, as shown in Fig. 3f. The OAm ligand peak is located at wavenumbers of $1200-1400 \mathrm{~cm}^{-1}$ of the $\mathrm{C}-\mathrm{H}$ bending mode, and $1600-$ $1800 \mathrm{~cm}^{-1}$ of $\mathrm{C}=\mathrm{C}$ stretching and $\mathrm{N}-\mathrm{H}$ bending mode. ${ }^{45}$ Raman spectra in Fig. 3f showed that the OAm ligand on AgNPs was gradually removed as the DUV irradiation time increased. However, in the case of the thermal treatment, the intensity peak corresponding to the OAm ligand was increased at an annealing temperature of $>150^{\circ} \mathrm{C}$, as shown in Fig. 3e. It can be considered that molten OAm molecules spontaneously desorbed on the NPs and moved to the top of the NP films or empty space formed by sintered NPs. Further, the remaining OAm cannot be easily removed owing to its high boiling point $(>300$ $\left.{ }^{\circ} \mathrm{C}\right)$. Consequently, the OAm ligand can be easily removed by DUV treatment at low temperature without a specific change in the AgNP shapes and the generation of undesirable products, resulting in a significant improvement of the electrical performances.

In the case of PVP-AgNPs, (Fig. 4a-d), the decrease in the distance between AgNPs and the increase in the AgNP size were accompanied by the PVP ligand removal and the partial nanowelding via DUV treatment, while the thermal-treated PVPAgNPs were aggregated by partial sintering at an annealing temperature greater than $120{ }^{\circ} \mathrm{C}$ (Fig. S5 in the ESI $\dagger$ ). As with OAm-AgNPs, thermally treated PVP-AgNPs exhibited large grain growth above $180{ }^{\circ} \mathrm{C}$ (Fig. 4b), as well as the subsequent formation of many voids owing to film shrinkage. Fig. 4e and $\mathrm{f}$ show the Raman spectra of thermal and DUV treatment for AgNPs with PVP ligands, respectively. As the annealing temperature and DUV irradiation time increased, the peak at $2935 \mathrm{~cm}^{-1}$ of the $\mathrm{C}-\mathrm{H}$ stretching mode corresponding to PVP ligands markedly decreased. ${ }^{46}$ Because DUV treatment enabled
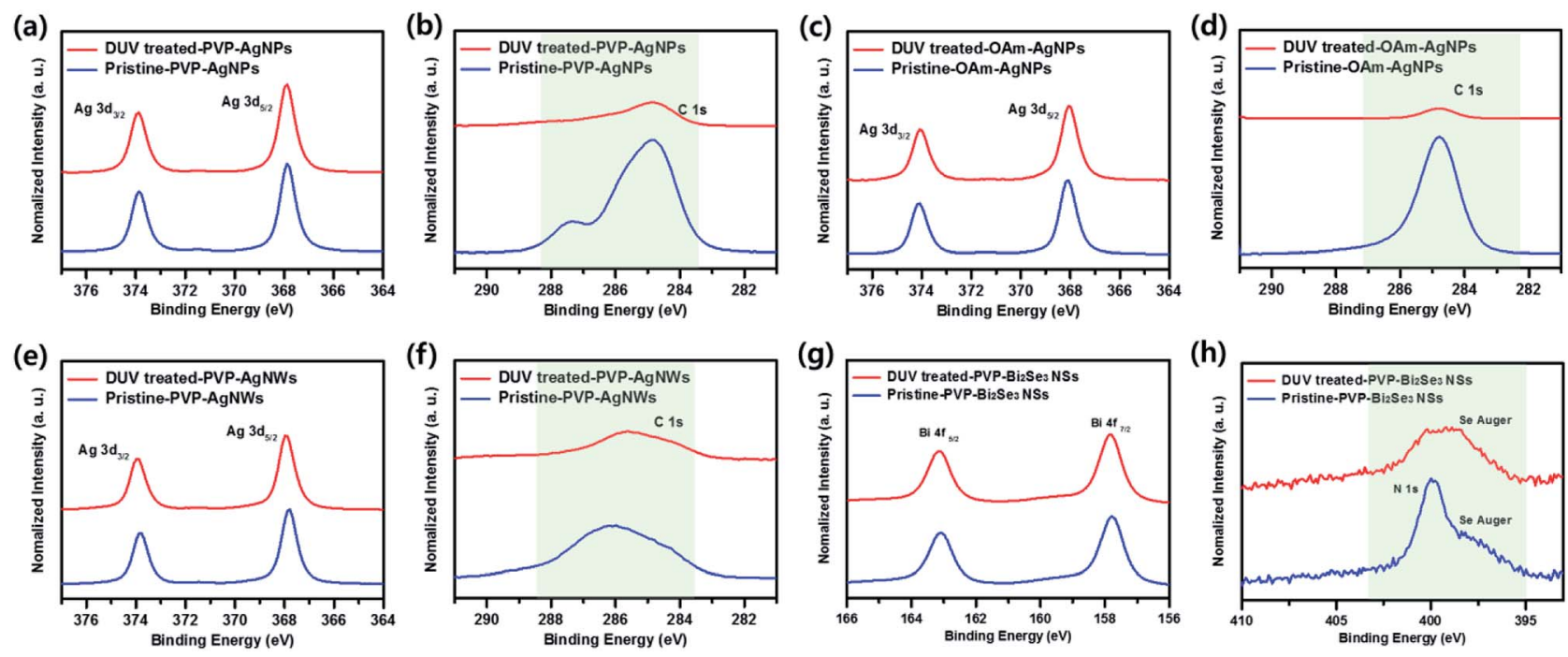

Fig. 5 X-ray photoelectron spectroscopy analysis of $C$ 1s (normalized to Ag 3d) and N 1s (normalized to Bi 4f) peaks for the before and after DUV treatment. ((a and d) PVP-AgNPs, (b and e) OAm-AgNPs, (c and f) PVP-AgNWs. (g and h) PVP-Bi $\mathrm{Se}_{3} \mathrm{NSs}$ ). 
the degradation of the $\mathrm{C}-\mathrm{H}, \mathrm{C}=\mathrm{O}$, even the $\mathrm{C}-\mathrm{C}$ bond by sigmasigma* or pi-pi* transition, a polymeric hydrocarbon chain was converted into short and volatile components that will be easily evaporated into the atmosphere. Therefore, the percolation conduction of AgNPs with PVP ligands could be improved by removing the potential barrier of organic ligands on metal nanomaterials using photochemical activation, resulting in enhanced charge-carrier transfer between nanomaterials. The facile removal of organic ligands with photochemical treatment could be further implemented for the fabrication of various solution-processed electronic devices with nanomaterials, such as thin-film transistors, solar cells, light emitting diodes, and sensors.

To demonstrate the removal of organic ligands more clearly, X-ray photoelectron spectra (XPS) of pristine and photochemical treated nanomaterial films were obtained. The $\mathrm{C} 1 \mathrm{~s}$ or $\mathrm{N} 1 \mathrm{~s}$ peaks were normalized with respect to $\mathrm{Ag} 3 \mathrm{~d}$ or $\mathrm{Bi} 4 \mathrm{f}$ intensities, respectively. As shown in Fig. 5, S6 and S7 (ESI†), the pristine samples without photochemical treatment exhibit large amounts of carbon or nitrogen contents from organic surfactant. In contrast, the carbon or nitrogen contents are significantly reduced after photochemical treatment, which clearly indicates that the organic ligands are removed by DUV irradiation at relatively low temperature (room temperature).

\section{Conclusions}

In this study, we confirmed that DUV treatment is an efficient method for the removal of the high-energy barrier polymeric or aliphatic organic ligands on the nanomaterials regardless of their morphology, such as NPs, NWs, and NSs. Although the NSs required additional excimer treatment to achieve the electronic properties, all of the DUV-treated materials exhibited much higher conductivities than the conductivities of thermally annealed samples. The thermally annealed AgNPs (PVP and $\mathrm{OAm}$ ) showed the significant aggregation of sintered particles by the removal of ligands and thermal shrinkage, which cause void generation within AgNP films $\left(>180^{\circ} \mathrm{C}\right)$, whereas the DUVtreated AgNPs were sintered by nano-welding without significant film shrinkage. In particular, the DUV treated of PVPAgNPs for 90 min $\left(50-60{ }^{\circ} \mathrm{C}\right)$ resulted in a sheet resistance of $0.54 \Omega \square^{-1}$, while thermally treated PVP-AgNP had a sheet resistance of $7.5 \mathrm{k} \Omega \square^{-1}$ at a similar annealing temperature of $60{ }^{\circ} \mathrm{C}$. The Raman spectrum showed the additional evidence for the efficient removal of organic ligands, which indicates that the DUV-treatment is more effective than heat treatment, even at low temperature. In addition, the removal of organic ligands was confirmed more clearly from the reduction of the carbon content, as shown in XPS spectra. In conclusion, the photochemical treatment of nanomaterials is an efficient processing strategy for low-temperature-processed nanomaterial matrixes for next-generation flexible and wearable electronic devices.

\section{Conflicts of interest}

There are no conflicts to declare.

\section{Acknowledgements}

This research was partially supported by the Chung-Ang University Research Grants in 2017, and by the National Research Foundation of Korea (NRF) grant funded by the Korean government (No. 2017R1C1B2005254).

\section{References}

1 B. Cho, J. Yoon, M. G. Hahm, D.-H. Kim, A. R. Kim, Y. H. Kahng, S.-W. Park, Y.-J. Lee, S.-G. Park, J.-D. Kwon, C. S. Kim, M. Song, Y. Jeong, K.-S. Nam and H. C. Ko, J. Mater. Chem. C, 2014, 2, 5280-5285.

2 X. Hu, X. Zhang, L. Liang, J. Bao, S. Li, W. Yang and Y. Xie, Adv. Funct. Mater., 2014, 24, 7373-7380.

3 Q. Hua, J. Sun, H. Liu, R. Bao, R. Yu, J. Zhai, C. Pan and Z. L. Wang, Nat. Commun., 2018, 9, 244.

4 J. Jang, B. G. Hyun, S. Ji, E. Cho, B. W. An, W. H. Cheong and J.-U. Park, NPG Asia Mater., 2017, 9, e432.

5 D. Kim, Y. Fu, S. Kim, W. Lee, K. H. Lee, H. K. Chung, H. J. Lee, H. Yang and H. Chae, ACS Nano, 2017, 11, 19821990.

6 S. Wang, J. Xu, W. Wang, G. N. Wang, R. Rastak, F. MolinaLopez, J. W. Chung, S. Niu, V. R. Feig, J. Lopez, T. Lei, S. K. Kwon, Y. Kim, A. M. Foudeh, A. Ehrlich, A. Gasperini, Y. Yun, B. Murmann, J. B. Tok and Z. Bao, Nature, 2018, 555, 83-88.

7 M. G. Kim, M. G. Kanatzidis, A. Facchetti and T. J. Marks, Nat. Mater., 2011, 10, 382-388.

8 C. Zhong, C. Duan, F. Huang, H. Wu and Y. Cao, Chem. Mater., 2011, 23, 326-340.

9 M. R. Niazi, R. Li, E. Qiang Li, A. R. Kirmani, M. Abdelsamie, Q. Wang, W. Pan, M. M. Payne, J. E. Anthony, D. M. Smilgies, S. T. Thoroddsen, E. P. Giannelis and A. Amassian, Nat. Commun., 2015, 6, 8598.

10 T. Sakanoue and H. Sirringhaus, Nat. Mater., 2010, 9, 736740.

11 M. K. Choi, J. Yang, K. Kang, D. C. Kim, C. Choi, C. Park, S. J. Kim, S. I. Chae, T. H. Kim, J. H. Kim, T. Hyeon and D. H. Kim, Nat. Commun., 2015, 6, 7149.

12 J. H. Heo, M. H. Lee, H. J. Han, B. R. Patil, J. S. Yu and S. H. Im, J. Mater. Chem. A, 2016, 4, 1572-1578.

13 Y. L. Rao, A. Chortos, R. Pfattner, F. Lissel, Y. C. Chiu, V. Feig, J. Xu, T. Kurosawa, X. Gu, C. Wang, M. He, J. W. Chung and Z. Bao, J. Am. Chem. Soc., 2016, 138, 6020-6027.

14 J. You, L. Meng, T. B. Song, T. F. Guo, Y. M. Yang, W. H. Chang, Z. Hong, H. Chen, H. Zhou, Q. Chen, Y. Liu, N. De Marco and Y. Yang, Nat. Nanotechnol., 2016, 11, 75-81.

15 W. Nie, H. Tsai, R. Asadpour, J.-C. Blancon, A. J. Neukirch, G. Gupta, J. J. Crochet, M. Chhowalla, S. Tretiak, M. A. Alam, H.-L. Wang and A. D. Mohite, Science, 2015, 347, 522-525.

16 X. Wang, F. Sun, Y. Duan, Z. Yin, W. Luo, Y. Huang and J. Chen, J. Mater. Chem. C, 2015, 3, 11397-11405. 
17 W. Ke, G. Fang, Q. Liu, L. Xiong, P. Qin, H. Tao, J. Wang, H. Lei, B. Li, J. Wan, G. Yang and Y. Yan, J. Am. Chem. Soc., 2015, 137, 6730-6733.

18 Y. S. Rim, H. Chen, X. Kou, H. S. Duan, H. Zhou, M. Cai, H. J. Kim and Y. Yang, Adv. Mater., 2014, 26, 4273-4278.

19 M. Akin, R. Bongartz, J. G. Walter, D. O. Demirkol, F. Stahl, S. Timur and T. Scheper, J. Mater. Chem., 2012, 22, 11529.

20 R. M. Arán-Ais, F. J. Vidal-Iglesias, J. Solla-Gullón, E. Herrero and J. M. Feliu, Electroanalysis, 2015, 27, 945-956.

21 S. Yu, C. Hwang, D. J. Kim, J. Park, S. Kim, J. Hong, J. An, T. H. Lee and M.-G. Kim, Thin Solid Films, 2018, 661, 46-52.

22 A. Nag, M. V. Kovalenko, J. S. Lee, W. Liu, B. Spokoyny and D. V. Talapin, J. Am. Chem. Soc., 2011, 133, 10612-10620.

23 D. Li, C. Wang, D. Tripkovic, S. Sun, N. M. Markovic and V. R. Stamenkovic, ACS Catal., 2012, 2, 1358-1362.

24 Z. Liu, M. Shamsuzzoha, E. T. Ada, W. M. Reichert and D. E. Nikles, J. Power Sources, 2007, 164, 472-480.

25 C.-H. Hwang, J.-P. Park, M.-Y. Song, J.-H. Lee and I.-W. Shim, Bull. Korean Chem. Soc., 2011, 32, 2207-2211.

26 B. Ingham, T. H. Lim, C. J. Dotzler, A. Henning, M. F. Toney and R. D. Tilley, Chem. Mater., 2011, 23, 3312-3317.

27 J. You, H. Ziruo, Y. Yang, Q. Chen, M. Cai, T.-B. Song, C.-C. Chen, S. Lu, L. Yongsheng, H. Zhou and Y. Yang, ACS Nano, 2014, 8, 1674-1680.

28 Z. Y. Chen, D. Liang, G. Ma, G. S. Frankel, H. C. Allen and R. G. Kelly, Corros. Eng., Sci. Technol., 2013, 45, 169-180.

29 N. C. Anderson, M. P. Hendricks, J. J. Choi and J. S. Owen, J. Am. Chem. Soc., 2013, 135, 18536-18548.

30 P. R. Brown, D. Kim, R. R. Lunt, N. Zhao, M. G. Bawendi, J. C. B. Grossman and V. Bulovic, ACS Nano, 2014, 8, 58635872 .

31 D. H. Webber and R. L. Brutchey, J. Am. Chem. Soc., 2012, 134, 1085-1092.

32 C. Hwang, J. An, B. D. Choi, K. Kim, S.-W. Jung, K.-J. Baeg, M.-G. Kim, K. M. Ok and J. Hong, J. Mater. Chem. C, 2016, 4, 1441-1447.
33 G. Jiang, X. Li, M. Lan, T. Shen, X. Lv, F. Dong and S. Zhang, Appl. Catal., B, 2017, 205, 532-540.

34 J. Kim, M. G. Kim, J. Kim, S. Jo, J. Kang, J. W. Jo, W. Lee, C. Hwang, J. Moon, L. Yang, Y. H. Kim, Y. Y. Noh, J. Y. Jaung, Y. H. Kim and S. K. Park, Sci. Rep., 2015, 5, 14520.

35 Y. H. Kim, J. S. Heo, T. H. Kim, S. Park, M. H. Yoon, J. Kim, M. S. Oh, G. R. Yi, Y. Y. Noh and S. K. Park, Nature, 2012, 489, 128-132.

36 D. Tsvetanova, R. Vos, G. Vereecke, T. N. Parac-Vogt, F. Clemente, K. Vanstreels, D. Radisic, T. Conard, A. Franquet, M. Jivanescu, D. A. P. Nguyen, A. Stesmans, B. Brijs, P. Mertens and M. M. Heyns, J. Electrochem. Soc., 2011, 158, H785.

37 Y. Sun, B. Mayers, T. Herricks and Y. Xia, Nano Lett., 2003, 3, 955-960.

38 J. Perelaer, A. W. M. de Laat, C. E. Hendriks and U. L. Schubert, J. Mater. Chem., 2008, 18, 3209-3215.

39 M. Chen, Y.-G. Feng, X. Wang, T.-C. Li, J.-Y. Zhang and D.-J. Qian, Langmuir, 2007, 23, 5296-5304.

40 Z. Lin, Y. Chen, A. Yin, Q. He, X. Huang, Y. Xu, Y. Liu, X. Zhong, Y. Huang and X. Duan, Nano Lett., 2014, 14, 6547-6553.

41 D. E. Xu, J. B. Kim, M. D. Hook, J. P. Jung and M. Mayer, J. Alloys Compd., 2018, 731, 504-514.

42 L. Mo, D. Liu, W. Li, L. Li, L. Wang and X. Zhou, Appl. Surf. Sci., 2011, 257, 5746-5753.

43 H. J. Hwang, K. H. Oh and H. S. Kim, Sci. Rep., 2016, 6, 19696. 44 K. Mallikarjuna, H.-J. Hwang, W.-H. Chung and H.-S. Kim, RSC Adv., 2016, 6, 4770-4779.

45 M. M. Al-Shalalfeh, A. T. Onawole, T. A. Saleh and A. A. AlSaadi, Mater. Sci. Eng., C, 2017, 76, 356-364.

46 R. G. Snyder, S. L. Hsu and S. Krimm, Spectrochim. Acta, 1978, 34A, 395-406. 